\title{
A Review of Tactile Information: Perception and Action Through Touch
}

\author{
Qiang Li, Oliver Kroemer, Zhe Su, Filipe Veiga, Mohsen Kaboli and Helge Ritter
}

\begin{abstract}
Tactile sensing is a key sensor modality for robots interacting with their surroundings. These sensors provide a rich and diverse set of data signals that contain detailed information collected from contacts between the robot and its environment. The data is however not limited to individual contacts and can be used to extract a wide range of information about the objects in the robots environment as well as the robots own actions during the interactions.

In this paper, we provide an overview of tactile information and its applications in robotics. We present a hierarchy consisting of raw, contact, object, and action levels to structure the tactile information, with higher-level information often building upon lower-level information. We discuss different types of information that can be extracted at each level of the hierarchy. The paper also includes an overview of different types of robot applications and the types of tactile information that they employ.

The paper concludes with a discussion of tactile-based computational framework and future tactile applications which are still beyond current robot's capabilities.

Index Terms-Tactile Sensing, Interactive Perception, Tactile Controller, Grasping, In-hand Manipulation, Whole Body Manipulation, Locomotion, Tool Manipulation, Human Robot Interaction
\end{abstract}

\section{INTRODUCTION}

Touch is an important sensing modality for robots physically interacting with their environment. Tactile sensing provides robots with a rich set of diverse signals based on contacts between the robot and its environment. These signals provide the robot with information about the objects in its environment as well its interactions with these objects, e.g., if an object begins to slip or if the robot has achieved a sturdy foothold. This information is crucial for performing a variety of tasks in a robust and reliable manner, including dexterous manipulation, locomotion, and human-robot interactions.

However, to use tactile sensing, robots first need to extract the relevant information from the sensor signals. We present an overview of the different types of tactile information that can be extracted from tactile signals. This information ranges from low-level forces at individual contact points to feedback for selecting complex actions based on previous interactions. We propose structuring the information in a hierarchy consisting of contact-, object-, and action-level information.

Qiang Li is with CITEC, Bielefeld University,Germany.

Oliver Kroemer is with Carnegie Mellon University (CMU), USA.

Zhe Su is with the University of Southern California (USC), USA

Filipe Veiga is with Computer Science and Artificial Intelligence Laboratory (CSAIL), Massachusetts Institute of Technology (MIT), USA.

Mohsen Kaboli is with IAS, Technical University of Munich (TUM) and the BMW Research,Germany.

Helge Ritter is with CITEC, Bielefeld University,Germany

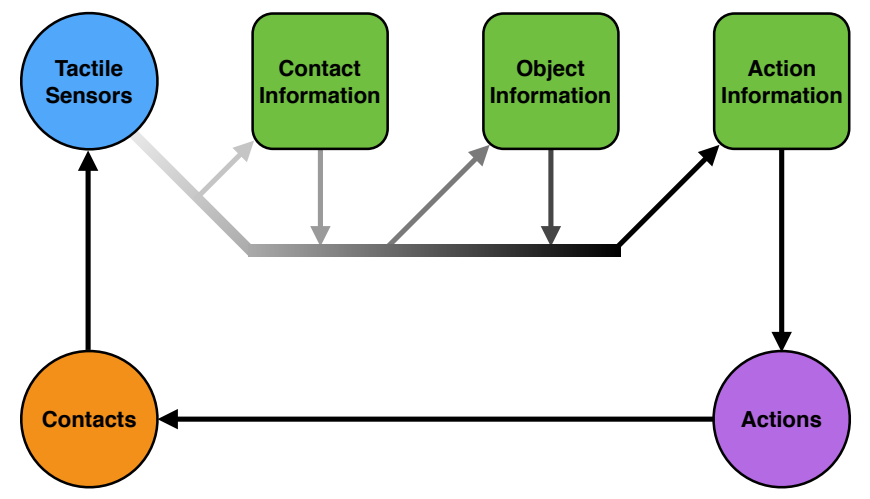

Fig. 1. Perception-action loop for tactile information extraction and control.

Higher-level information tends to build on the information extracted from lower levels as illustrated in Fig. 1. While the contact level contains information from individual contact sites, the object level contains information regarding objects as a whole, often acquired from multiple contacts. The action level refers to information pertaining to the robots actions used to interact with the environment, which often builds upon contact- and object-level information. As a contact-based sensing modality, actions are also important for extracting useful information from the interactions. All three of these levels build upon the sensor-level tactile signals. For each level of the hierarchy, we describe the different types of information that are commonly extracted at that level. We also explain how robot applications employ tactile information from across the different levels.

A number of previous tactile review papers focused on the sensor's hardware [1]-[4], and discussed specific application domains, e.g., grasping and in-hand manipulation [5][7]. Recently Luo et al. [8] showed the newest progress on tactile perception. Akihiko and Christopher [9] reviewed the progress on tactile manipulation and put the highlight on the vision-based tactile sensors and its applications. In this paper, we propose a computational framework to structure tactile perception and actions. We highlight the different types of tactile information and propose a hierarchy for structuring the information. We also explain how various robot applications employ tactile information from across the hierarchy.

The paper is organized as follows. An overview of tactile signals is introduced in Sec. II. Then we explain in detail tactile information at each level of the hierarchy in Sec III through V. In Sec. VI, we briefly discuss analytical and data-driven methods for computing the tactile information. In 
Sec. VII, the applications are discussed with regard to how tactile information is used in rich tactile-based tasks, including tactile exploration, grasping, in-hand manipulation, tool manipulation, locomotion and human robot interaction. We conclude the paper with a discussion of open challenges and future research directions for tactile perception and control.

\section{Sensor-LeVel Tactile Signals}

A robot with the sense of touch can acquire information about its surroundings through physical interactions. The sensors are located under the surface of artificial skin [10], [11], [3] and provide the robot with a wide range of signals. In this section, we briefly discuss common types of sensory signals that will form the basis of the computational framework proposed in this paper. We also discuss tactile sensor coverage-how sensors are distributed on the body and how spatial resolution are often selected depending on the given task and the sensors' placement on the robot.

\section{A. Normal and Tangential Force}

The most common type of tactile signal is the contact force. Although the tactile sensors are based on the different physical principle, the output (tactile image, voltage) from the raw sensor measurement can be "calibrated" as contact force. Traditionally, the contact force is divided into two components: normal force and shear or tangential force. Normal force is the component orthogonally applied to the contact surface, and tangential force is the component applied across the surface, e.g., friction. Most tactile sensors are able to measure normal force [12], [13]. Some sensors can however directly measure the full 3D force [14]-[18]. The majority of tactile sensors are composed of arrays of sensing elements [3], [19]-[21], and each element in the array is known as a taxel. Each taxel provides a local force estimate corresponding to its activation. In addition to contact force, the contact torque may also be estimated by the sensor array [22], [23] using a trained neural network.

\section{B. Vibration}

Mechanical vibrations are another fundamental type of tactile signals that is often used to detect contact or slip events between contact surfaces [24]-[26]. When we use a hammer to strike a nail [27], we can feel the vibration via our skin's mechanoreceptors [28]. A robot can similarly detect vibrations using a dynamic tactile sensor. Vibration signals from tactile sensors can be used to detect whether slip occurs between the sensors and the directly contacted object or between a grasped object and another object. This is possible because the vibrations in the latter case propagate additionally through the grasped object and the tactile patterns perceived by the sensors in the two situations are therefore different [29]. Like the fast afferents in human skin, vibration-based sensing is not as useful in static contacts situations, as motion is required to induce vibrations [30].

\section{Thermal}

Thermal tactile sensing allows a robot to measure the temperature of an object via touching [31], [32]. It mimics the thermal sensing of humans, which are able to perceive temperatures between $5^{\circ}$ and $45^{\circ}$ [33]. Thermal sensing can also be combined with heating elements to allow the robot to estimate the thermal conductivity of an object [21]. The differences in thermal conductivity can then be used to distinguish between different object materials [34], [35]. For example, metals transfer heat faster than most plastics and rubbers. Determining the thermal state of the contacted object is also very important to decide the next actions. e.g. a robot can estimate a cup's temperature to autonomously reason whether it is safe for serving. In the teleoperation scenario, thermal sensing can help the operator to judge the types of remote objects [36].

\section{Pretouch Proximity}

Pretouch is the ability to detect objects and their surfaces before contacting with them. A pretouch sensor can provide a robot with the relative geometrical relation to an object [37] , which is valuable for the robot's planners [38], [39] and controllers [40], [41], [42]. With the distance, the robot can roughly estimate the object's shape and position before making contact. Depending on the underlying technology, the pretouch sensors may also provide additional information such as color and optical flow [43]. Pretouch sensors can also provide the robot with better predictions regarding when the contact will be made and thus prevent the robot from making contact at high speeds. The principle technology of pretouch sensors varies, and they can be based on cutaneous infrared [44], audio [45], or optical sensing [46]. In this manner, pretouch sensing blurs the line between different sensor modalities.

\section{E. Sensor Coverage}

Besides the type of tactile signals, another important characteristic of tactile sensing is the distribution of sensors throughout the robot's body. Body parts covered with high spatial resolution tactile sensors can be used for contact recognition and fine motor control, while parts with lower resolution of sensing can be used for simple contact detection and control. Most tactile skin designs for robots are inspired by the human

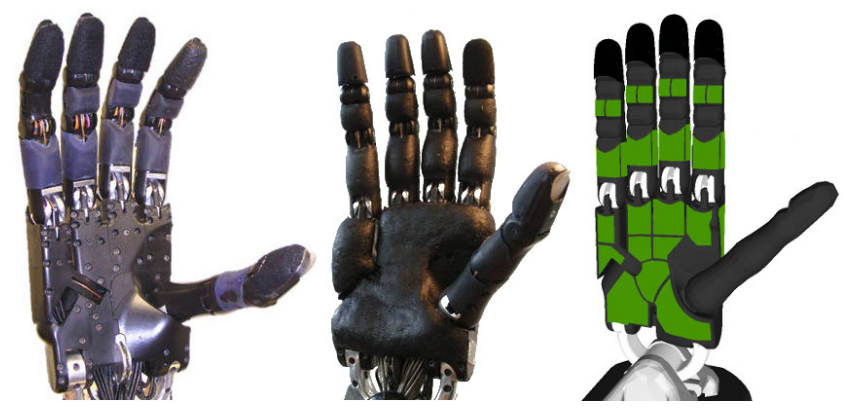

Fig. 2. A Shadow Dexterous Hand (left) that has been covered with a tactile skin (middle) in order to provide tactile information at several locations (highlighted greep regions on the right rendering). 


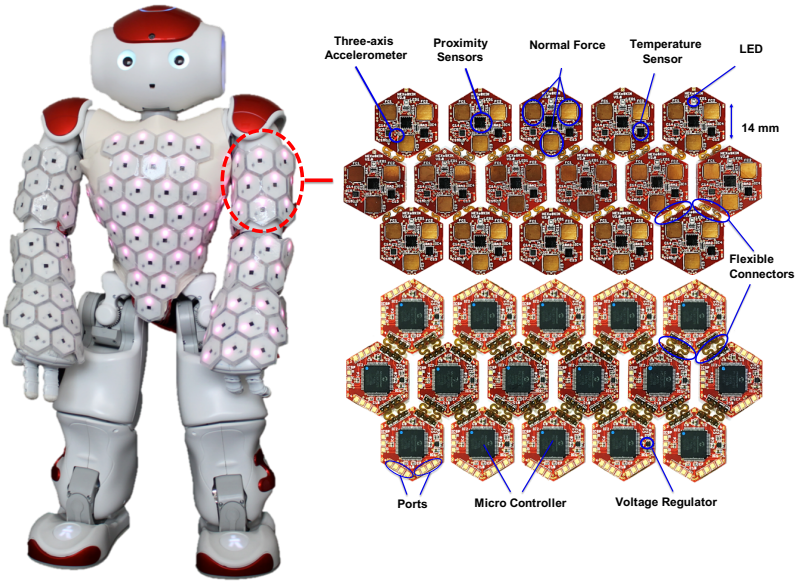

Fig. 3. The NAO humanoid robot covered with a multimodal artificial robotic skin that provides vibration, temperature, force and proximity information [47].

tactile perception system, in which neuroscientists have found that the sensitive tactile receptors are unevenly distributed in the whole body. For example, hands have higher tactile spatial resolutions than other parts to facilitate dexterous manipulation [48].

Many robots' hands are equipped with tactile sensors, especially at the fingertips [23], [49], [50]. An example of a robot hand with tactile sensors on the finger tips phalanges and palm [51] can be seen in Fig. 2. High spatial and temporal resolutions provide hands with rich tactile information for performing complex tasks, e.g. exploring unknown objects, tool use, and in-hand manipulation.

Tactile sensors can also be embedded in other robot body parts, such as arms [52], [53], torso (Fig. 3), legs [54] and feet [55]. Contact information on these parts are closely associated with tasks involving whole-body manipulations, human robot interaction and locomotion. The density of the sensing elements is coarser on these parts because the sampling and processing of large areas of taxels is challenging from a hardware design and communication perspective [3]. The tactile sensors in load bearing parts of the body are often designed in a more robust way to withstand the larger forces needed to grasp heavy objects [56] and support the robot's weight [57] during walking tasks. Tactile sensors are normally located on the surfaces of regularly shaped links. However, flexible and stretchable sensors have been developed to cover body parts with complex shapes and curved surfaces, [58], [59], as well as the robot's joints [60].

\section{CONTACT-LEVEL INFORMATION}

The basic function of tactile sensing is to provide a robot with information about the contact sites, such as local geometries, forces, material properties, and contact events (Fig. 4). In this section, we discuss the type of contact information and how the contact information is extracted from raw tactile signals. This information forms the basic building blocks for the object-level information (Sec.IV) and is widely used for controlling the robot (Sec.V).

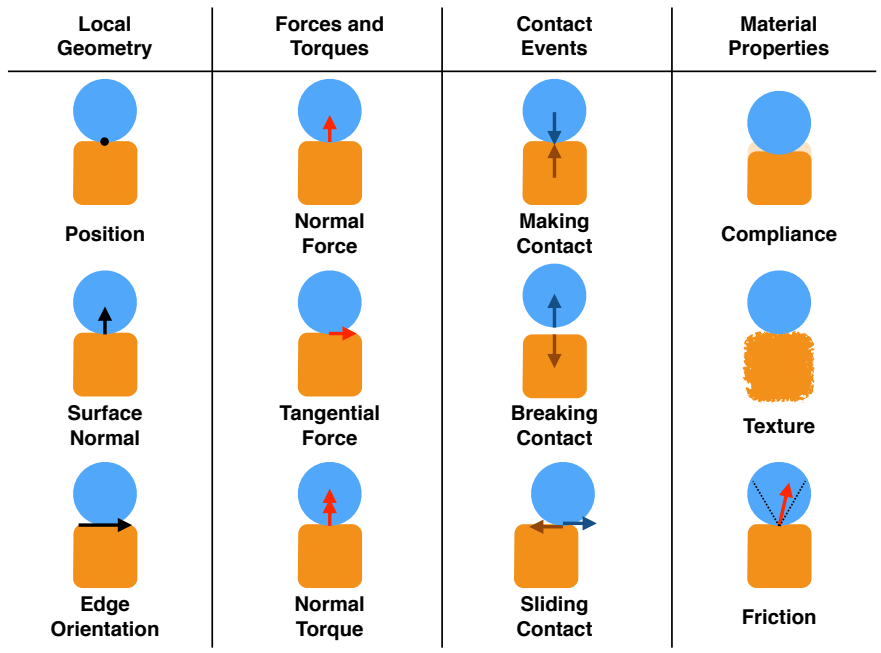

Fig. 4. Examples of different types of contact-level information that can be extracted from the sensor-level tactile signals.

\section{A. Contact Geometry}

Tactile sensing is widely used to estimate the local geometry of a contact area including the contact position, normal, and curvature. Many tactile-related tasks rely on precise measurements of the contact position and normal direction, e.g., to compute grasp stability and manipulability [61]. Given a calibrated sensor array [62], the contact position in the tactile sensor frame is computed as the pressure-weighted center of the activated taxels and then mapped to the robot's Cartesian frame using the robot's forward kinematics [63], [64]. Using the same principle, the contact normal direction is estimated as the pressure-weighted normal direction of the activated taxels. The position and normal provide a first order approximation of the local surface patch, with the normal direction defining the tangent plane at the contact point.

Tactile sensors can also be used for estimating higher surface derivatives at the contact point, e.g., the surface curvature [65], [66]. Given a tactile sensor with high spatial resolution, the contact surface curvature can be measured based on the relative movement between the sensor and an unknown object when sliding or rolling occurs [65]. For sensors with lower resolutions, the contact surface can be approximated with a second order polynomial equation [61], [66]. The curvature at the contact point is approximated by a local parameterized surface matrix that can be estimated through active tactile exploration.

Tactile image processing is another way to extract contact geometry of a small region. Machine-learning [67], [68] and traditional image based approaches [63], [69]-[71] are widely used to estimate the contact position or feature position for different types of object by detecting salient structures such as edges, corners and small protrusions. For example, a pronounced curvature on an object's surface will appear as a line in the tactile image. The slope of the line, relative to the tactile sensor, can be estimated by the principle component of the image blob [72]. The 3D direction of the edge is then estimated by combining the line's slope with the robot's 
forward kinematic model.

\section{B. Force and Torque}

Contact forces and torques are directly measured by several tactile sensors [12], [20], [21], [43], [67]. Some sensors only provide the normal force while others also provide tangential forces to give the full $3 \mathrm{D}$ force vector. Estimating forces at contacts is important, as it is the transfer of forces that allows the robot to physically interact with objects in its environment. When employing a point contact model, the contact force can be estimated from the contact pressures of all activated taxels [20], [63] or from the sensor's overall deformation [43], [67].

Different approaches have been proposed for mapping raw tactile pressure values to the contact forces and torques [73] [75]. One example is to model the mapping as a linear function [76], [77]. The drawback of this approach is that it does not work well for sensors that have strong hysteresis or damping properties due to the skin material [2]. For such skin materials, other advanced machine learning algorithms are more suitable. For example, locally weighted projection regression and artificial neural networks can be used to learn the nonlinear relation [25], and Gaussian processes can be used to compensate for the nonlinear hysteresis effects of sensors [78]. Finite element methods have also been used to model the nonlinear functions and learn the model parameters [73].

\section{Contact Events}

Contact events refer to discrete changes in the contact state between two objects, which include making, breaking, sliding and rolling contacts. Contact events often correspond to subgoals and errors when interacting with the environment. For example, breaking and making contact between a foot and the ground is the goal of each step in a walking task, while a sliding foothold is usually considered to be an error.

The transition between contact and no contact is a nearinstantaneous event that results in a discontinuous interaction model. It is therefore important that the robot can detect the events quickly and reliably. A common way to detect the contact is to compare the measured tactile signals with a given threshold [60], [79], which is estimated from previous contact experiences. Another method is to use dynamic tactile sensors [80], which compute the vibration features to distinguish contact from no contact. Using this approach, it is possible to detect the contacts not only between the robot's skin and the environment but also between a grasped object and the environment [81].

In addition to transitions from no contact to contact, the robot may also experience the change from a static contact to a sliding contact. Since lots of approaches assume no slip and slip often corresponds to errors and is difficult to control, robust detection of slip is a crucial research topic. In practice, gross sliding is usually preceded by an incipient slip event, wherein only some low-pressure regions of the contact patch begin to slide [80]-[83]. That is to say, the outer regions on a fingertip begin slipping while the central region, where more pressure is applied, does not slip [63],
[84]. Hence, detecting incipient slip is a key capability for avoiding gross sliding from happening. Incipient slip can be detected with high-frequency tactile feedback (normally $>1 \mathrm{kHz}$ ) using frequency-domain analysis approaches [79], [80], [83], [85]. Other approaches include learning from data and using image processing techniques, e.g., using a Hebbian network [86], using optical flow from a fingertip camera [87], or using random forests to predict slip from multi-modal tactile information [88].

Robots can additionally differentiate between different types of slip, e.g., determining whether the gross sliding is rotational or translational [24], [25], [43], [71], [89]. For such classification, neural networks are trained to classify time-series tactile pressure data or visual features for vision-based tactile sensors [25], [89].

\section{Material Properties}

The interactive nature of touch allows a robot to estimate object's material properties which may not be easily extracted visually. Researchers have defined 15 different properties to present the object's surface [90], and these properties can be reduced to 5 important dimension, namely stiffness, friction, surface texture thermal conductivity and adhesion. Stiffness can be measured by the robot pressing into the object with a specified contact force and measuring the resulting displacement of the contact point [91]-[94]. Similarly the coefficient of static friction can be estimated by measuring the normal and tangential forces when incipient slip occurs [95], [96].

Texture information is useful for differentiating among materials, as well as detecting blemishes and smooth surfaces. To achieve good classification result, a robot will often slide the tactile sensor across the surface and observe the resulting vibrations and time series signals [97]. Classifiers, such as $\mathrm{kNN}$, ANNs, and SVMs, can then be trained to classify the different textures [98]-[101]. Better classification performance can be achieved by employing multiple sliding motions with varying velocities and directions [102].

Thermal conductivity is another useful material property. While the temperature of an object is estimated by a thermal sensor, the thermal conductivity of the material is estimated by the transfer rate of thermal energy [21], [103]. Given that materials conduct heat at different rates, the detected transfer rate can also be used to classify different materials [35], [104].

\section{OBJECT-LEVEL INFORMATION}

Many tasks involve interacting with or manipulating unknown objects. To perform these tasks reliably, the robot needs to estimate the state and properties of the manipulated objects. Tactile sensors can be used to acquire a wide range of object-level information via raw sensor values or by combining contact-level information during interactions. Inspired by human exploration actions for determining object properties [30], we illustrate several common actions to extract necessary object-level information in Fig. 5. 


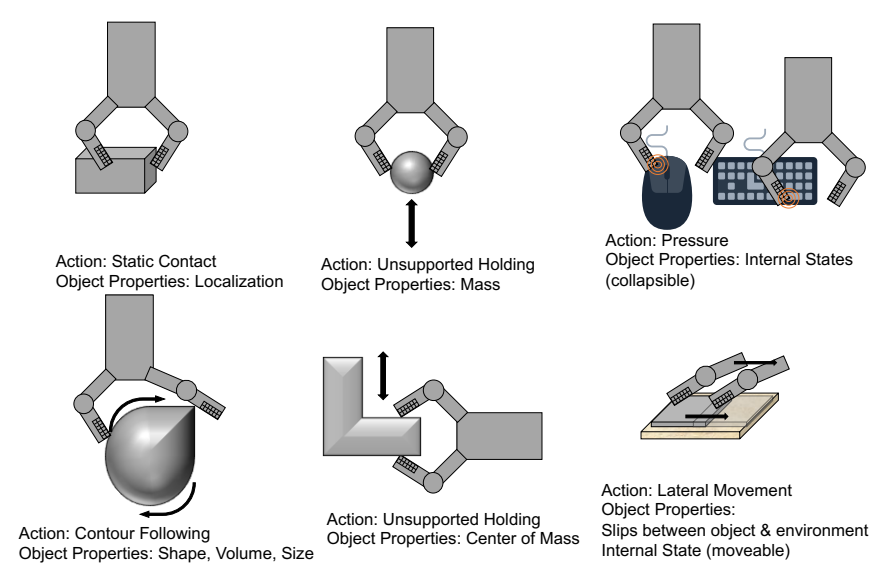

Fig. 5. Several actions for which different types of object-level information are necessary for the actions execution.

\section{A. Object Localization}

A robot needs to accurately estimate the pose of an object in order to precisely manipulate it to a desired location. Localizing an object using tactile feedback only is not a trivial task. Approaches are commonly based on filtering theory [105][108]. These approaches use the measured contact positions and normal of the object as input, and probabilistic models of the object's pose are then updated over time to capture the uncertainty of the estimation over multiple interactions [107].

In addition to accuracy, the efficiency of estimation is also important especially for tactile exploration tasks. To this end, optimization approaches can be used to select informative actions and explore the most uncertain pose space [109]-[112]. It is often assumed that the object is static and not affected by the exploration [105], [109], [110], [113]. Researchers have also proposed methods for tracking an object's pose while it is being moved [106], [114].

\section{B. Shape}

The shape of an object refers to its global geometry. Shape information is often needed to plan interactions with objects. For example, a robot with a two fingered gripper needs to find opposing surfaces of an object for grasping. The shape can be reconstructed by measuring the positions and normals of surface patches [115]. Employing tactile sensing is especially useful for estimating the object's shape in visually occluded regions [116]-[118]. The tactile sensors can thus play a complementary role to vision sensors for updating the object's model .

By touching the object at one single location, tactile sensors only estimate the local shape of relatively small regions, so multiple contacts are needed in order to obtain the global shape. This procedure is time consuming, and extensive efforts have therefore focused on optimizing touch sequences to reduce the uncertainty of the estimated shape and improve the model as quickly as possible [119]-[121]. The shapes are often represented by Gaussian processes to model the uncertainty [120] or by fitting geometrical models to the tactile point clouds [112].

\section{Mass and Dynamics}

While an object is grasped or manipulated, it acts as an additional payload for the robot. The mass and CoM are then required for the accurate dynamics model of the new system composed by the robot arm and hand plus the grasped object [61]. When grasping an object, the robot should place its hand near the object's center of mass to avoid large torques. Conversely, the robot can estimate the center of mass by slightly lifting the object and observing the torques. Correctly estimating the mass and center of mass, allows the robot to avoid improper contact force by using the estimated information to adapt the grasp force.

The estimation of the object's mass and CoM requires an interactive control procedure. Different action strategies and learning approaches have been proposed for estimating them and also the object's inertial matrix [122]-[126]. While grasping a heavy object, a robot can estimate the object's mass parameters based on the force and torque measurements from the wrist [122], [127]. For light objects, tactile estimates of the fingertip forces during simple lifting actions can be used to localize the center of mass of unknown objects [123], [124]. For an object that is too large to grasp, a robot can estimate its mass parameters by tipping it and stabilizing it in a different posture [125], [128].

\section{Contents of Containers}

The interactive nature of touch allows tactile sensing to estimate properties of objects that would otherwise be latent. In addition to the mass properties of an object, a robot may also determine the contents of container objects. For example, a robot may determine if a non-rigid container is full or empty, as well as open or closed, by squeezing it and observing the resulting tactile signals [129].

In addition to squeezing action, a robot can shake a container to estimate the amount of material inside or the material properties of the contents [102], [130], [131]. One example approach estimated the viscosity of a liquid within a container by using a learned Gaussian process model and selecting different shaking behaviors to actively acquire the best estimate [130].

\section{ACTION-LEVEL INFORMATION}

For most applications, robots need to execute sequences of actions to finish more complex tasks [132], e.g., grasping, transporting, releasing for pick-and-place tasks or executing a sequence of steps for walking tasks. Tactile sensing can be used to compute action-level information for performing and monitoring the complex task at all stages (see Fig. 6). Action level information may be computed from contactlevel [25], [79], [88] and object-level [106], [114], [123], [124] information as well as directly from tactile signals [26].

\section{A. Action Selection and Initialization}

Tactile data from previous actions and interactions can be used to select the next action and initialize its parameters. Robots can use previous actions to explore the objects and 


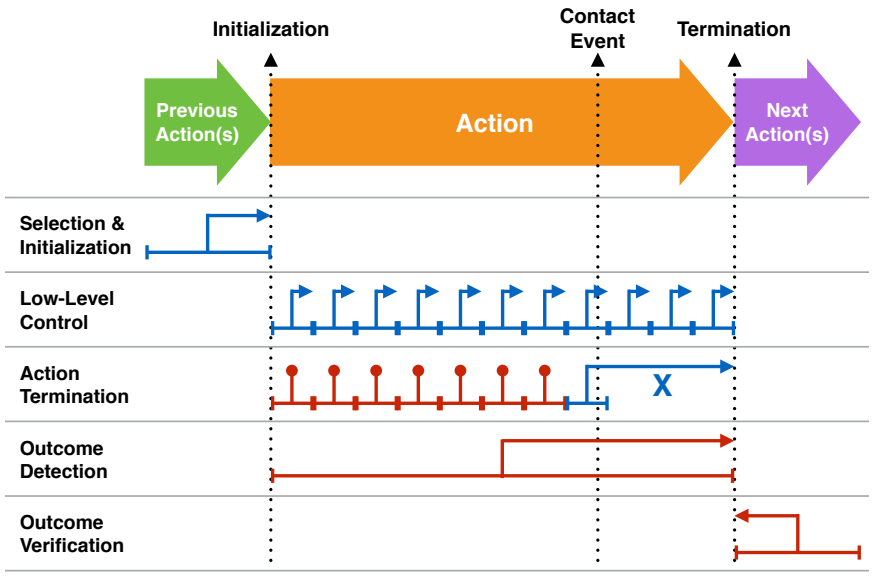

Fig. 6. The large arrows at the top show a sequence of actions' execution. In the lower part, horizontal baselines indicate the time window when the tactile information is being extracted. The arrows indicate the time point when the action is monitored and the action is performed or adapted. Action selection and initialization uses tactile signals from the previous interaction to select and set the parameters of the current action. Low-level control uses the sensory data from each time step to select the next low-level control input during the action execution. Action termination monitors the action at each time step and either continues the action (red) or terminates it (blue) if a specific contact even has been detected. Action outcome detection then determine if the executed action was successful, or which type of error occured, based on the tactile signals acquired during the action execution Action outcome verification uses an additional action to generate more tactile signals to determining the outcome.

extract object-level information, then use the updated object model for initializing the current action [106], [115], [133].

The exploratory actions themselves are selected to acquire additional information based on the current belief for the object's properties [110]. For example, when the robot has acquired a partial 3D model of an object based on previous tactile feedback, then the next grasp should be selected to explore locations where the model is still uncertain [118], [120]. Similarly, the robot may select other exploration parameters e.g. different normal forces and velocities for a series of finger sliding movements in order to better recognize an object [97].

Action selection is also used for recovering from failed grasps and other actions. When a robot's grasp attempt fails, the robot can use the data from the failure to extract latent object properties and adapt the grasp for the next attempt accordingly [134], [135]. The resulting regrasping strategy thus has a higher likelihood of succeeding by exploiting the tactile information to select the next grasp.

\section{B. Tactile Feedback for Low-level Control}

Once a robot starts to execute an action, it can employ continuous tactile feedback to control the interaction by computing suitable motor commands [136]. For this computation, contact-level information [25], [79], [88], object-level information [106], [114], [123], [124] or raw tactile signals [26], [137] are required.

Tactile servoing uses feedback from tactile features to control the pose of the robot end-effector and maintain a certain amount of pressure while interacting with objects with various material properties [72], [77], [123], [138]-[140]. This approach allows the robot to explore the unknown object, actively extract object's features [141], [142] and cope with the geometry and material uncertainties of the contacted objects. It is also useful for tracing the edges of objects [72]. In servoing tasks, action and perception are tightly coupled as the robot needs to continuously adapt its actions to the current tactile signals.

In addition to continuous servoing control, the robot may also compute actions to respond to certain contact-level events, e.g., incipient slip. Reacting to these events requires quick reflexes. In many cases, incipient slip detection triggers an increase in the normal force exerted by the controller [24], [83]. Rather than waiting for the slip event, the robot may also learn to predict these events in advance using tactile sensing [25], [88].

\section{Action Termination}

Action termination allows a robot to determine if the current action should be continued or terminated, such that the robot can then switch to another action. Terminating an inappropriate action early allows the robot to reduce the negative effects of colliding with objects or other errors, as well as avoiding errors when a goal is reached earlier than expected.

To perform action termination, the robot continuously monitors the tactile signals and learns a binary classifier. Given the model classifier and the current tactile data, the robot can determine if the action should be continued or terminated [143]. Another way to decide whether an action should be terminated is to learn a model of the expected sensory signals [144]. The robots compare the predictive output of this model to the actual sensor values during the action execution [140], [145]. The action is then terminated if the deviation from the expected model output is too large.

\section{Action Outcome Detection}

Actions are not guaranteed to always succeed, even when robust tactile feedback is used in the control loop. Therefore, it is important for the robot to determine that the goal is reached, and no error occurred during execution. Outcome detection can be formulated as a classification problem where the classifier has a binary output indicating if the action was successful or not [146]. The robot may also attempt to determine the specific type of error if one occurred. The outcome classification may also be achieved with a probabilistic approach that computes the probability of a successful outcome [135]. For the input, the robot may use the entire time series tactile signal from the action execution. However, often only the final frame is used as it tends to be highly informative for determining the outcome [147], [148].

Outcome detection has been used for evaluating grasp stability. In this case, classifiers or probabilistic models are used to determine if a grasp was successful before attempting to lift the object [135], [146]-[150]. Rather than continuing the planned sequence of actions, a detected failure may trigger a regrasping action or a replanning strategy [135]. Outcome detection can thus serve a similar role to action initialization, but for the subsequent action. 
Outcome detection is also important for providing the robot with additional information for future executions of the action. The robot can use outcome detection as feedback for learning actions from experience [151], [152]. Successful outcomes give greater rewards and thus encourage similar action executions in the future.

\section{E. Action Outcome Verification}

Many outcomes are ambiguous given only the observed tactile signals during the action execution. A robot therefore needs to use interactive perception to disambiguate these situations. By applying an additional action, and observing the resulting tactile signals, a robot can estimate more reliably the outcome of the previous action. For example, a robot may attempt to perturb a screw sideways to verify that it was correctly inserted into a hole [153]. A robot may also attempt to lift an object to verify that a grasp was successful [134]. Similar with direct outcome detection, the outcome verification can be posed as a classification problem. However, the input data is provided by the tactile signals from the following actions. Outcome verification requires extra time and effort to perform the subsequent action, and it may change the state as a result, but it also provides useful information for determining the outcome.

\section{TACTILE COMPUTATION: ANALYTICAL AND DATA-DRIVEN MODELS}

Tactile computation approaches can generally be divided into two groups-analytical or data-driven.

Analytical approaches exploit physics-based models to compute tactile information [66], [80], [96], [107], [120], [127], [77]. From the descriptions of tactile information in Sec III through $\mathrm{V}$, it is clear that many of the processed signals have a clear physical meaning. Higher-level information, such as object properties and action commands, are computed from the raw tactile signals or from contact information by accumulating interactions and using physical models. By utilizing the principles of physics to create models of the environment, human operators can easily understand the robots' perception procedure and its decision-making processes. However, these models normally rely on structured interactions and accurate feedback signals. If precise information of the interactions is not available and more complex tasks are being considered, simplified assumptions about the interactions must be taken to facilitate the design of the models.

Another approach is to employ data-driven methods to compute and process the tactile information implicitly [74], [135], [144], [154], [155]. These approaches learn mappings from raw sensory signals, or lower-level features, to high-level object properties and action commands. Supervised, unsupervised, and even reinforcement learning methods can be used to learn suitable features for a variety of tactile tasks. Hierarchical representations, such as neural networks, are often used to learn multiple levels of features. The learned intermediary features are generally not interpretable by humans. Data-driven methods generally do not require a precise model of the interaction and they tend to avoid brittle assumptions. Flexible representations allow the robot to adapt the learned model to the specific task based directly on data. It is often easier to provide data from contact-based interactions than to predefine an accurate analytical model.

\section{APPLICATIONS OF TACTILE INFORMATION}

Rich tactile information provides lots of possibility for performing tactile-relevant tasks, which include: tactile exploration, grasping, in-hand manipulation, locomotion, tool manipulation and human robot interaction. In this section, we summarize the relation among tactile information, computation method and applications in the table I. The table elements can be read as "in X application, $X$ tactile information is studied with X method". We discuss and give examples that how robots can compute and use contact-, object-, and actionlevel information within these complex task domains.

\section{A. Tactile Exploration}

Tactile exploration is an effective way to extract properties of an unknown object through touch [141], [142]. Humans use many exploration procedures to obtain knowledge about objects [30] - lateral motions, pressure, enclosure, contour following, object's part motion tests, and affordance tests. Inspired by these findings, many tactile-based exploration methods have been developed for robots to estimate important parameters including contact level information such as local geometry [66] and material properties [97], as well as object level information such as shape [156], [157] and mass information [123]. In addition to the analytical approaches, recently some researchers were proposing the data-driven approaches to learn the contact geometry and object's properties [75] [159] using large tactile dataset.

Another representative application of tactile sensing in exploration is tactile servoing control. This is mainly computing the action level information to maintain the desired contact pattern defined by contact level information considering single [72], [138] or multiple contact areas [158]. In the example of single contact [72], [138], a tactile planar array is assembled on the end-effector of the arm. The desired tactile pattern is the specified contact position and force. The tactile array explores the object's surface by implement sliding and rolling actions. The goal of the action information is to minimize the deviation of the tactile pattern. This approach can also be used to control a robot hand for exploration [158]. This controller was using multi-contact area on hand and exploring the surface of an unknown object to improve the robot hands grasping capability.

\section{B. Grasping}

Grasping is one of the most widely researched aspects of robot manipulation [198], as it provides a robot with control over the grasped objects and it is a common prerequisite for tool usage. Similar to tactile exploration, tactile grasping is also an important method to extract object properties. Some researchers extracted the contact level information [81], [91] and object level information [89], [105], [110], [126] using 
TABLE I

A SUMMARY FOR TACTILE INFORMATION AND COMPUTATION APPROACHES IN APPLICATIONS

\begin{tabular}{|c|c|c|c|c|}
\hline & computation approach & Contact level & Object level & Action level \\
\hline Tactile Exploration & $\begin{array}{c}\text { analytical models } \\
\text { data-driven }\end{array}$ & $\begin{array}{c}{[66],[97]} \\
{[75]}\end{array}$ & $\begin{array}{c}{[123],[156],[157]} \\
{[159]}\end{array}$ & $\begin{array}{c}\text { [72], [138], [158] } \\
{[160][161]}\end{array}$ \\
\hline Grasping & $\begin{array}{c}\text { analytical models } \\
\text { data-driven }\end{array}$ & $\begin{array}{c}{[81],[91]} \\
{[164],[165]}\end{array}$ & $\begin{array}{c}{[89],[105],[110],[126]} \\
{[166],[167]}\end{array}$ & $\begin{array}{c}{[162],[163]} \\
{[134],[168]-[170]}\end{array}$ \\
\hline In-hand Manipulation & $\begin{array}{c}\text { analytical models } \\
\text { data-driven }\end{array}$ & $\begin{array}{l}{[171]} \\
{[154]}\end{array}$ & $\begin{array}{l}{[172]} \\
{[178]} \\
\end{array}$ & $\begin{array}{c}\text { [173], [174], [137], [175], [176], [177] } \\
{[179],[151]}\end{array}$ \\
\hline Tool Manipulation & $\begin{array}{c}\text { analytical models } \\
\text { data-driven }\end{array}$ & $\begin{array}{c}{[180]} \\
{[29]}\end{array}$ & $\begin{array}{l}{[181]} \\
{[139]}\end{array}$ & {$[182][183],[143],[153]$} \\
\hline Locomotion & $\begin{array}{c}\text { analytical models } \\
\text { data-driven }\end{array}$ & $\begin{array}{c}{[184],[95],[185],[186]} \\
{[189]-[191]}\end{array}$ & $\begin{array}{l}- \\
-\end{array}$ & {$[187],[188]$} \\
\hline Human Robot Interaction & $\begin{array}{c}\text { analytical models } \\
\text { data-driven }\end{array}$ & $\begin{array}{l}- \\
-\end{array}$ & $\begin{array}{l}- \\
-\end{array}$ & $\begin{array}{c}\text { [192] [193], [194], [195] } \\
\text { [196] [197] } \\
\end{array}$ \\
\hline
\end{tabular}

analytical methods, and others computed them with data driven approaches [164]-[167].

Tactile sensing has also been used for analytical grasp controllers [162], [163], and for data-driven grasp synthesis, grasp outcome detection, and re-grasping [134], [148], [169]. Analytical approaches rely on accurate contacts position, normal direction, and force estimates to compute a grasp posture that maximizes the grasp quality metric [158], [199]. For data-driven approaches, the robot uses contact and object information from previous grasps to predict grasp qualities and compute regrasp postures [110], [134], [148], [169], [200].

Once the object has been grasped, tactile feedback can be used to control the contact forces [201], [202] and detect incipient slip [25], [81], [83]. By detecting or predicting incipient slip using tactile data, the robot can automatically increase its grip force to avoid gross slip without having to explicitly estimate the object-finger friction coefficients [203].

\section{In-hand Manipulation}

In-hand manipulation involves using the dexterity of the robots hand to change the state of a grasped object. For this task, some work focused on the computation of contact and object level information [171], [172], [154], [178], but the majority of work was to study the tactile controllers to relocate the grasped objects. The controllers have been performed with either multifingered robot hand [23], [173], [177], [204]-[207] or grippers [208]-[210].

For local repositioning while maintaining contact, the object is precisely grasped then moved with the robot's fingertips [173]. Tactile sensing is used to estimate the contact and object information and actively control the contacts between the fingers and the object [174], [176]. Alternatively, tactile sensing can also be used to directly learn a mapping from the contact sensor information to the robotic fingers desired joint velocities [151], [179] in a data-driven way.

In order to move the object further [211], robots need use finger gaiting to switch between different grasps while maintaining the object in hand. To this end, the fingers need tactile sensing to detect breaking and contacting with the object to effectively walk the hand around the objects surface. For dexterous hands, researchers have proposed using humaninspired finger gaiting strategies [175]. In order to imitate the human, demonstrations of gaiting behaviors with tactile signals can be acquired by fitting the human subject's hands with tactile finger caps [212].

\section{Tool Manipulation}

Tool usage is a core aspect of many manipulation tasks [213]. A key aspect of employing tactile sensing in tool manipulation is that the task contacts are between an object and the held tool. The contacts are therefore not directly on the tactile sensors, unless the tool is itself instrumented [180].

Tactile sensing can be used to detect contacts on tools, localize tooltips and other important parts of the manipulated tools [29], monitor the progress of tasks and detect manipulation failures. Tactile feedback can also be used to estimate and maintain the orientation and forces at the contact points to perform the compliance control tasks. Tactile sensing can also be employed in a dual-arm setup to estimate the kinematic parameters of a grasped tool (1 D.O.F) [181].

Tactile sensing is also used for controlling and using unknown tools. The main challenge is that no kinematic or dynamic manipulation model can be directly used to compute the tactile action information for a given task. To this end, data driven methods [182] become a valid approach to compute the action command implicitly. There is more challenge interactive control task-multi-stages contact interaction. For example, a peg may slide freely or become jammed during an insertion task. Tactile sensing allows the robot to interactively detect these different types of contact modes [143] and plan recovery actions accordingly [153].

\section{E. Locomotion}

Tactile sensing is not only useful for manipulation tasks, but also for locomotion. Ground vehicles and walking robots, such as humanoids, quadruped, and hexapod robots, need to use contacts with the environment to move around. Tactile sensing provides to them with estimates of the state of the robot and the environment terrain [95], [214] during locomotion. For example, wheeled robots can use tactile sensing to monitor their contact with the ground [189]-[191]. Vibration signals from microphones or accelerometers in the wheels can be used for determining the type of terrain.

Maintaining balance is a key part of both standing and walking. To maintain balance, the robot needs to use tactile sensing to estimate its contact locations for support [95], as 
well as detect obstacles and other perturbations that may cause it to lose balance [187]. The tactile sensing can also be used for learning a tactile-motor mapping for standing [186]. Walking requires deliberately planning and controlling gaits, which consist of multiple phases [215]. The transitions between phases are often triggered by contact events, such as the heel contacts with the ground. Tactile sensing has also been used to study where to place a foot [188]. By estimating the type of terrain, a robot can generate appropriate gaits and switch to a suitable leg controller.

\section{F. Human Robot Interaction}

In addition to interacting with inanimate objects and terrain, robots also need to physically interact with humans. Human robot interaction (HRI) applications span a wide range of different tasks including intuitive programming [192], putting on clothing [196], handing over objects [216], safe interaction with collaborative robots [217], [218] and shaving [193]. During these tasks, the robot need to ensure that the interaction forces are safe and adapt to the humans body.

In the context of HRI, contact and object level information is computed with the similar way like tactile exploration and grasping. Recent new progress is focusing on inferring the humans latent state and intentions from sensory feedback [219]-[221]. For example, during a handover, the robot can employ vision and tactile feedback to determine when the human has a suitable grip and is ready to accept the object [194]. Similarly, when performing collaborative tasks such as carrying large objects, the robot measures interactive forces and torques through the force/torque sensor on the wrists for performing the task and react accordingly [195].

In addition to performing tasks, human robot interaction can also be used to teach robots new skills from demonstrations [222]. In this manner, the robot can be directly guided by a human using force controllers [223]. Physical interactive corrections can also be used to refine robots skill executions through touch [197].

\section{CONCLUSION AND FUTURE WORK}

Tactile sensing is an important sensing modality as it allows robots to estimate properties of objects and interactions, and it provides feedback for adapting the robots' executed actions. In this paper, we discussed the main types of information that can be acquired using tactile signals as well as different types of applications in which this information can be used. We explained how tactile sensing can be used to acquire data related to individual contacts, objects, and skills. These different types of information can be extracted from the sensor signals using analytical techniques or data-driven approaches. The information extracted from these various types of methods can then be used for a wide range of different applications. The proposed categorization of tactile perception approaches is thus applicable to various task domains.

Our review of tactile information focused on the different types of estimation problems that robots must overcome, rather than the specific types of methods used to address these problems. In this manner, we provide a structure for this research field that can be applied both to past work as well as future research. Although we have made great strides as a research community, none of the presented problems should be considered as solved.

In addition to developments for the individual challenges that we discussed, we also expect to see more approaches that collect contact- and object- level information while performing actions for a given task. Methods for contact- and objectlevel information currently tend to employ purely exploratory skills, e.g., stroking and prodding [141], [158] or basic manipulations, e.g. simple grasping and lifting [105], [110], [124]. Similarly, action-level methods often assume that object and contact information are already provided or it is fixed and therefore does not need to be explicitly represented [113], [120]. Future work on tactile sensing will also explore deeper how analytical and data-driven approaches can be combined more efficiently. Current methods often focus either on analytical [66], [80] or data driven approaches [135], [154], [179]. By combining these two approaches will allow robots to work efficiently by exploiting their prior knowledge and adapting to novel situations autonomously.

\section{REFERENCES}

[1] H. R. Nicholls and M. H. Lee, "A survey of robot tactile sensing technology," International Journal of Robotics Research, vol. 8, no. 3, pp. 3-30, 1989.

[2] M. H. Lee and H. R. Nicholls, "Review article tactile sensing for mechatronicsa state of the art survey," Mechatronics, vol. 9, no. 1, pp. 1-31, 1999.

[3] R. S. Dahiya, G. Metta, M. Valle, and G. Sandini, "Tactile sensingfrom humans to humanoids.," IEEE Transactions on Robotics, vol. 26, no. 1, pp. 1-20, 2010.

[4] M. R. Cutkosky, R. D. Howe, and W. R. Provancher, "Force and tactile sensors," Springer Handbook of Robotics, pp. 455-476, 2008.

[5] Z. Kappassov, J.-A. Corrales, and V. Perdereau, "Tactile sensing in dexterous robot hands," Robotics and Autonomous Systems, vol. 74, pp. 195-220, 2015.

[6] J. Tegin and J. Wikander, "Tactile sensing in intelligent robotic manipulation-a review," Industrial Robot: An International Journal, vol. 32, no. 1, pp. 64-70, 2005.

[7] R. D. Howe, "Tactile sensing and control of robotic manipulation," Advanced Robotics, vol. 8, no. 3, pp. 245-261, 1993.

[8] S. Luo, J. Bimbo, R. Dahiya, and H. Liu, "Robotic tactile perception of object properties: A review," Mechatronics, vol. 48, pp. 54-67, 2017.

[9] A. Yamaguchi and C. G. Atkeson, "Recent progress in tactile sensing and sensors for robotic manipulation: can we turn tactile sensing into vision?," Advanced Robotics, pp. 1-13, 2019.

[10] Z. Stojiljkovic and J. Clot, "Integrated behavior of artificial skin," IEEE Transactions on Biomedical Engineering, no. 4, pp. 396-399, 1977.

[11] E. Cheung and V. Lumelsky, "Development of sensitive skin for a 3d robot arm operating in an uncertain environment," in Proceedings, 1989 International Conference on Robotics and Automation, pp. 1056-1061, IEEE, 1989.

[12] C. Schurmann, R. Haschke, H. Ritter, et al., "A modular high-speed tactile sensor for human manipulation research," in IEEE World Haptics Conference (WHC), pp. 339-344, IEEE, 2011.

[13] Y. Zhang, "Sensitivity enhancement of a micro-scale biomimetic tactile sensor with epidermal ridges," Journal of Micromechanics and Microengineering, vol. 20, no. 8, p. 085012, 2010.

[14] Á. S. Tar, G. G. Cserey, and J. Veres, "Sensor device,” Dec. 62016. US Patent 9,513,178.

[15] H. Liu, K. C. Nguyen, V. Perdereau, J. Bimbo, J. Back, M. Godden, L. D. Seneviratne, and K. Althoefer, "Finger contact sensing and the application in dexterous hand manipulation," Autonomous Robots, vol. 39, no. 1, pp. 25-41, 2015.

[16] Y. Zhang, Y. Mukaibo, and T. Maeno, "A multi-purpose tactile sensor inspired by human finger for texture and tissue stiffness detection," in IEEE International Conference on Robotics and Biomimetics (ROBIO), pp. 159-164, IEEE, 2006 
[17] W.-C. Choi, "Polymer micromachined flexible tactile sensor for threeaxial loads detection," Transactions on Electrical and Electronic Materials, vol. 11, no. 3, pp. 130-133, 2010.

[18] T. P. Tomo, A. Schmitz, W. K. Wong, H. Kristanto, S. Somlor, J. Hwang, L. Jamone, and S. Sugano, "Covering a robot fingertip with uskin: A soft electronic skin with distributed 3-axis force sensitive elements for robot hands," IEEE Robotics and Automation Letters, vol. 3, no. 1, pp. 124-131, 2017.

[19] B. Ward-Cherrier, N. Pestell, L. Cramphorn, B. Winstone, M. E. Giannaccini, J. Rossiter, and N. F. Lepora, "The tactip family: Soft optical tactile sensors with 3d-printed biomimetic morphologies," Soft Robotics, vol. 5, no. 2, pp. 216-227, 2018.

[20] N. Jamali, M. Maggiali, F. Giovannini, G. Metta, and L. Natale, "A new design of a fingertip for the icub hand," in IEEE/RSJ International Conference on Intelligent Robots and Systems (IROS), pp. 2705-2710, IEEE, 2015.

[21] N. Wettels, J. A. Fishel, and G. E. Loeb, "Multimodal tactile sensor," in The Human Hand as an Inspiration for Robot Hand Development, pp. 405-429, Springer, 2014.

[22] C. G.De Maria and S.Pirozzi, "Force/tactile sensor for robotic applications," Sensors and Actuators A: Physical, vol. 175, pp. 60-72, 2012.

[23] G. Palli, C. Melchiorri, G. Vassura, U. Scarcia, L. Moriello, G. Berselli, A. Cavallo, G. De Maria, C. Natale, S. Pirozzi, et al., "The dexmart hand: Mechatronic design and experimental evaluation of synergybased control for human-like grasping," The International Journal of Robotics Research, vol. 33, no. 5, pp. 799-824, 2014.

[24] C. Melchiorri, "Slip detection and control using tactile and force sensors," IEEE ASME Transactions on Mechatronics, vol. 5, no. 3, pp. 235-243, 2000.

[25] Z. Su, K. Hausman, Y. Chebotar, A. Molchanov, G. E. Loeb, G. S. Sukhatme, and S. Schaal, "Force estimation and slip detection/classification for grip control using a biomimetic tactile sensor," in IEEE-RAS 15th International Conference on Humanoid Robots (Humanoids), pp. 297-303, IEEE, 2015.

[26] M. Meier, G. Walck, R. Haschke, and H. J. Ritter, "Distinguishing sliding from slipping during object pushing," in IEEE/RSJ International Conference on Intelligent Robots and Systems (IROS), pp. 5579-5584, IEEE, 2016.

[27] T. Izumi and Y. Hitaka, "Hitting from any direction in 3-d space by a robot with a flexible link hammer," IEEE Transactions on Robotics and Automation, vol. 13, no. 2, pp. 296-301, 1997.

[28] R. S. Johansson and J. R. Flanagan, "Coding and use of tactile signals from the fingertips in object manipulation tasks," Nature Reviews Neuroscience, vol. 10, no. 5, p. 345, 2009.

[29] A. Molchanov, O. Kroemer, Z. Su, and G. S. Sukhatme, "Contact localization on grasped objects using tactile sensing," in IEEE/RSJ International Conference on Intelligent Robots and Systems (IROS), pp. 216-222, IEEE, 2016.

[30] S. J. Lederman and R. L. Klatzky, "Hand movements: A window into haptic object recognition," Cognitive Psychology, vol. 19, no. 3 , pp. 342-368, 1987.

[31] D. Siegel, I. Garabieta, and J. Hollerbach, "An integrated tactile and thermal sensor," in IEEE International Conference on Robotics and Automation (ICRA), vol. 3, pp. 1286-1291, IEEE, 1986.

[32] J. Wade, T. Bhattacharjee, and C. C. Kemp, "A handheld device for the in situ acquisition of multimodal tactile sensing data," in IROS Workshop on See and Touch: 1st Workshop on multimodal sensor-based robot control for HRI and soft manipulation, 2015.

[33] S. J. Lederman and R. L. Klatzky, "Haptic perception: A tutorial," Attention, Perception, \& Psychophysics, vol. 71, no. 7, pp. 1439-1459, 2009.

[34] E. Kerr, T. M. McGinnity, and S. Coleman, "Material classification based on thermal propertiesa robot and human evaluation," in IEEE International Conference on Robotics and Biomimetics (ROBIO), pp. 1048-1053, IEEE, 2013.

[35] T. Bhattacharjee, J. Wade, and C. C. Kemp, "Material recognition from heat transfer given varying initial conditions and short-duration contact," in Robotics: Science and Systems (RSS), Georgia Institute of Technology, 2015.

[36] D. G. Caldwell and C. Gosney, "Enhanced tactile feedback (teletaction) using a multi-functional sensory system," in [1993] Proceedings IEEE International Conference on Robotics and Automation, pp. 955-960, IEEE, 1993.

[37] K. Koyama, M. Shimojo, T. Senoo, and M. Ishikawa, "High-speed high-precision proximity sensor for detection of tilt, distance, and contact," IEEE Robotics and Automation Letters, vol. 3, no. 4, pp. 3224 3231, 2018.
[38] E. Cheung and V. Lumelsky, "Motion planning for robot arm manipulators with proximity sensing," in Proceedings. 1988 IEEE International Conference on Robotics and Automation, pp. 740-745, IEEE, 1988.

[39] L. Zlajpah and B. Nemec, "Kinematic control algorithms for online obstacle avoidance for redundant manipulators," in IEEE/RSJ international conference on intelligent robots and systems, vol. 2, pp. 1898-1903, IEEE, 2002.

[40] Onrobot, "rg2-ft-gripper." https://onrobot.com/en/products/ rg2-ft-gripper, 2019. [Online; accessed 7-Nov-2019].

[41] K. Hsiao, P. Nangeroni, M. Huber, A. Saxena, and A. Y. Ng, "Reactive grasping using optical proximity sensors," in 2009 IEEE International Conference on Robotics and Automation, pp. 2098-2105, IEEE, 2009.

[42] K. Koyama, Y. Suzuki, A. Ming, and M. Shimojo, "Integrated control of a multi-fingered hand and arm using proximity sensors on the fingertips," in 2016 IEEE International Conference on Robotics and Automation (ICRA), pp. 4282-4288, IEEE, 2016.

[43] A. Yamaguchi and C. G. Atkeson, "Combining finger vision and optical tactile sensing: Reducing and handling errors while cutting vegetables," in IEEE-RAS 15th International Conference on Humanoid Robots (Humanoids), pp. 1045-1051, IEEE, 2016.

[44] D. Balek and R. Kelley, "Using gripper mounted infrared proximity sensors for robot feedback control," in Proceedings. 1985 IEEE International Conference on Robotics and Automation, vol. 2, pp. 282-287, IEEE, 1985

[45] L.-T. Jiang and J. R. Smith, "Seashell effect pretouch sensing for robotic grasping," in 2012 IEEE International Conference on Robotics and Automation, pp. 2851-2858, IEEE, 2012.

[46] D. Guo, P. Lancaster, L.-T. Jiang, F. Sun, and J. R. Smith, "Transmissive optical pretouch sensing for robotic grasping," in 2015 IEEE/RSJ International Conference on Intelligent Robots and Systems (IROS), pp. 5891-5897, IEEE, 2015.

[47] M. Kaboli and G. Cheng, "Robust tactile descriptors for discriminating objects from textural properties via artificial robotic skin," IEEE Transactions on Robotics, vol. 34, no. 4, pp. 985-1003, 2018.

[48] R. S. Johansson and A. Vallbo, "Tactile sensibility in the human hand: relative and absolute densities of four types of mechanoreceptive units in glabrous skin.," Journal of Physiology, vol. 286, no. 1, pp. 283-300, 1979.

[49] A. Schmitz, M. Maggiali, L. Natale, B. Bonino, and G. Metta, "A tactile sensor for the fingertips of the humanoid robot icub," in IEEE/RSJ International Conference on Intelligent Robots and Systems (IROS), pp. 2212-2217, IEEE, 2010.

[50] R. Koiva, M. Zenker, C. Schürmann, R. Haschke, and H. J. Ritter, "A highly sensitive 3d-shaped tactile sensor," in IEEE/ASME International Conference on Advanced Intelligent Mechatronics (AIM), pp. 10841089, IEEE, 2013.

[51] G. Buescher, M. Meier, G. Walck, R. Haschke, and H. J. Ritter, "Augmenting curved robot surfaces with soft tactile skin," in IEEE/RSJ International Conference on Intelligent Robots and Systems (IROS), pp. 1514-1519, IEEE, 2015.

[52] E. Dean-Leon, B. Pierce, F. Bergner, P. Mittendorfer, K. RamirezAmaro, W. Burger, and G. Cheng, "Tomm: Tactile omnidirectional mobile manipulator," in IEEE International Conference on Robotics and Automation (ICRA), pp. 2441-2447, IEEE, 2017.

[53] P. Mittendorfer, E. Yoshida, and G. Cheng, "Realizing whole-body tactile interactions with a self-organizing, multi-modal artificial skin on a humanoid robot," Advanced Robotics, vol. 29, no. 1, pp. 51-67, 2015.

[54] G. Cannata, M. Maggiali, G. Metta, and G. Sandini, "An embedded artificial skin for humanoid robots," in IEEE International Conference on Multisensor Fusion and Integration for Intelligent Systems (MFI), pp. 434-438, IEEE, 2008.

[55] K. Suwanratchatamanee, M. Matsumoto, and S. Hashimoto, "Haptic sensing foot system for humanoid robot and ground recognition with one-leg balance," IEEE Transactions on Industrial Electronics, vol. 58 , no. 8, pp. 3174-3186, 2011

[56] T. Mukai, M. Onishi, T. Odashima, S. Hirano, and Z. Luo, "Development of the tactile sensor system of a human-interactive robot ri-man," IEEE Transactions on robotics, vol. 24, no. 2, pp. 505-512, 2008.

[57] Y. Takahashi, K. Nishiwaki, S. Kagami, H. Mizoguchi, and H. Inoue, "High-speed pressure sensor grid for humanoid robot foot," in 2005 IEEE/RSJ International Conference on Intelligent Robots and Systems, pp. 3909-3914, IEEE, 2005.

[58] K. Noda, E. Iwase, K. Matsumoto, and I. Shimoyama, "Stretchable liquid tactile sensor for robot-joints," in IEEE International Conference on Robotics and Automation (ICRA), pp. 4212-4217, IEEE, 2010. 
[59] Y. Hirai, Y. Suzuki, T. Tsuji, and T. Watanabe, "Tough, bendable and stretchable tactile sensors array for covering robot surfaces," in IEEE International Conference on Soft Robotics (RoboSoft), pp. 276-281, IEEE, 2018.

[60] T. Bhattacharjee, A. Jain, S. Vaish, M. D. Killpack, and C. C. Kemp, "Tactile sensing over articulated joints with stretchable sensors," in IEEE World Haptics Conference (WHC), pp. 103-108, IEEE, 2013.

[61] R. M. Murray, Z. Li, S. S. Sastry, and S. S. Sastry, A mathematical introduction to robotic manipulation. CRC press, 1994.

[62] A. Del Prete, S. Denei, L. Natale, F. Mastrogiovanni, F. Nori, G. Cannata, and G. Metta, "Skin spatial calibration using force/torque measurements," in IEEE/RSJ International Conference on Intelligent Robots and Systems (IROS), pp. 3694-3700, IEEE, 2011.

[63] T. Nagatani, A. Noda, S. Hirai, et al., "What can be inferred from a tactile arrayed sensor in autonomous in-hand manipulation?", in IEEE International Conference on Automation Science and Engineering (CASE), pp. 461-468, IEEE, 2012.

[64] P. K. Allen, A. T. Miller, P. Y. Oh, and B. S. Leibowitz, "Integration of vision, force and tactile sensing for grasping," International Journal of Intelligent Machines, vol. 4, pp. 129-149, 1999.

[65] D. J. Montana, "The kinematics of contact and grasp," International Journal of Robotics Research, vol. 7, no. 3, pp. 17-32, 1988.

[66] N. Chen, R. Rink, and H. Zhang, "Local object shape from tactile sensing," in IEEE International Conference on Robotics and Automation (ICRA), vol. 4, pp. 3496-3501, IEEE, 1996.

[67] W. Yuan, S. Dong, and E. H. Adelson, "Gelsight: High-resolution robot tactile sensors for estimating geometry and force," Sensors, vol. 17, no. 12 , p. $2762,2017$.

[68] K. S. H. K. Pornthep Sarakona, Yuta Sakaib and S. Serikawaa, "Object shape and force estimation using deep learning and optical tactile sensor," in 6th IIAE International Conference on Intelligent Systems and Image Processing, 2018.

[69] R. Li, R. Platt, W. Yuan, A. ten Pas, N. Roscup, M. A. Srinivasan, and E. Adelson, "Localization and manipulation of small parts using gelsight tactile sensing," in 2014 IEEE/RSJ International Conference on Intelligent Robots and Systems, pp. 3988-3993, IEEE, 2014.

[70] A. M. Okamura, M. L. Turner, and M. R. Cutkosky, "Haptic exploration of objects with rolling and sliding," in IEEE International Conference on Robotics and Automation (ICRA), vol. 3, pp. 2485-2490, IEEE, 1997.

[71] S. Dong, W. Yuan, and E. H. Adelson, "Improved gelsight tactile sensor for measuring geometry and slip," in IEEE/RSJ International Conference on Intelligent Robots and Systems (IROS), pp. 137-144, IEEE, 2017.

[72] Q. Li, C. Schürmann, R. Haschke, and H. Ritter, "A control framework for tactile servoing," in Robotics: Science and Systems (RSS), 2013.

[73] A. Cirillo, P. Cirillo, G. De Maria, C. Natale, and S. Pirozzi, "Modeling and calibration of a tactile sensor for robust grasping," IFACPapersOnLine, vol. 50, no. 1, pp. 6843-6850, 2017.

[74] N. Wettels and G. E. Loeb, "Haptic feature extraction from a biomimetic tactile sensor: force, contact location and curvature," in IEEE International Conference on Robotics and Biomimetics (ROBIO), pp. 2471-2478, IEEE, 2011.

[75] P. Piacenza, S. Sherman, and M. Ciocarlie, "Data-driven superresolution on a tactile dome," IEEE Robotics and Automation Letters, vol. 3, no. 3, pp. 1434-1441, 2018.

[76] B. Navarro, P. Kumar, A. Fonte, P. Fraisse, G. Poisson, and A. Cherubini, "Active calibration of tactile sensors mounted on a robotic hand," in IEEE/RSJ International Conference on Intelligent Robots and Systems (IROS), IEEE/RSJ, 2015.

[77] Z. Su, J. Fishel, T. Yamamoto, and G. Loeb, "Use of tactile feedback to control exploratory movements to characterize object compliance," Frontiers in Neurorobotics, vol. 6, 2012.

[78] T. Horii, Y. Nagai, L. Natale, F. Giovannini, G. Metta, and M. Asada, "Compensation for tactile hysteresis using gaussian process with sensory markov property," in IEEE-RAS 15th International Conference on Humanoid Robots (Humanoids), pp. 993-998, IEEE, 2014.

[79] J. M. Romano, K. Hsiao, G. Niemeyer, S. Chitta, and K. J. Kuchenbecker, "Human-inspired robotic grasp control with tactile sensing," IEEE Transactions on Robotics, vol. 27, no. 6, pp. 1067-1079, 2011.

[80] M. R. Cutkosky and J. M. Hyde, "Manipulation control with dynamic tactile sensing," in International Symposium on Robotics Research (ISRR), vol. 117, 1993.

[81] B. Heyneman and M. R. Cutkosky, "Slip classification for dynamic tactile array sensors," International Journal of Robotics Research, vol. 35 , no. 4 , pp. 404-421, 2016.
[82] M. R. Tremblay and M. R. Cutkosky, "Estimating friction using incipient slip sensing during a manipulation task," in IEEE International Conference on Robotics and Automation (ICRA), pp. 429-434, IEEE, 1993.

[83] M. Schöpfer, C. Schürmann, M. Pardowitz, and H. Ritter, "Using a piezo-resistive tactile sensor for detection of incipient slippage," in International Symposium on Robotics (ISR) and German Conference on Robotics (ROBOTIK), pp. 1-7, VDE, 2010.

[84] V. A. Ho and S. Hirai, "Understanding slip perception of soft fingertips by modeling and simulating stick-slip phenomenon.," in Robotics: Science and Systems (RSS), 2011.

[85] S. Teshigawara, K. Tadakuma, A. Ming, M. Ishikawa, and M. Shimojo, "High sensitivity initial slip sensor for dexterous grasp," in IEEE International Conference on Advanced Robotics (ICAR), 2010.

[86] Y. Tada, K. Hosoda, and M. Asada, "Learn to grasp utilizing anthropomorphic fingertips together with a vision sensor," in IEEE/RSJ International Conference on Intelligent Robots and Systems (IROS), pp. 3323-3328, 2005

[87] A. Maldonado, H. Alvarez, and M. Beetz, "Improving robot manipulation through fingertip perception," in IEEE/RSJ International Conference on Intelligent Robots and Systems (IROS), pp. 2947-2954, IEEE, 2012.

[88] F. Veiga, J. Peters, and T. Hermans, "Grip stabilization of novel objects using slip prediction," IEEE Transactions on Haptics, vol. 11, no. 4 , pp. 531-542, 2018.

[89] M. Meier, F. Patzelt, R. Haschke, and H. J. Ritter, "Tactile convolutional networks for online slip and rotation detection," in International Conference on Artificial Neural Networks (ICANN), pp. 12-19, Springer, 2016.

[90] S. Inc, "Working with syntouch and the biotac toccare." https://www.syntouchinc.com/wp-content/uploads/2017/07/WP WorkingWithSynTouch_071017.pdf, 2019. [Online; accessed 7-Nov2019].

[91] H. Yussof, M. Ohka, A. R. Omar, and M. A. Ayub, "Determination of object stiffness control parameters in robot manipulation using a prototype optical three-axis tactile sensor," in IEEE Sensors, pp. 992995, IEEE, 2008.

[92] A. Drimus, G. Kootstra, A. Bilberg, and D. Kragic, "Classification of rigid and deformable objects using a novel tactile sensor," in IEEE International Conference on Advanced Robotics (ICAR), pp. 427-434, IEEE, 2011.

[93] T. Bhattacharjee, J. M. Rehg, and C. C. Kemp, "Inferring object properties with a tactile-sensing array given varying joint stiffness and velocity," International Journal of Humanoid Robotics, vol. 15, no. 01, p. 1750024, 2018.

[94] M. Kaboli, D. Feng, and G. Cheng, "Active tactile transfer learning for object discrimination in an unstructured environment using multimodal robotic skin," International Journal of Humanoid Robotics, vol. 15, no. 01, p. 1850001,2018

[95] T. Okatani, H. Takahashi, K. Noda, T. Takahata, K. Matsumoto, and I. Shimoyama, "A tactile sensor using piezoresistive beams for detection of the coefficient of static friction," Sensors, vol. 16, no. 5 , p. 718, 2016.

[96] K. Nakamura and H. Shinoda, "A tactile sensor instantaneously evaluating friction coefficients," in International Conference on Solid-State Sensors and Actuators, pp. 1402-1405, Springer, 2001.

[97] J. A. Fishel and G. E. Loeb, "Bayesian exploration for intelligent identification of textures," Frontiers in Neurorobotics, vol. 6, p. 4, 2012.

[98] M. Kaboli, R. Walker, G. Cheng, et al., "In-hand object recognition via texture properties with robotic hands, artificial skin, and novel tactile descriptors," in IEEE-RAS 15th International Conference on Humanoid Robots (Humanoids), pp. 1155-1160, IEEE, 2015.

[99] A. Drimus, M. B. Petersen, and A. Bilberg, "Object texture recognition by dynamic tactile sensing using active exploration," in IEEE International Symposium on Robot and Human Interactive Communication (RO-MAN), pp. 277-283, IEEE, 2012.

[100] O. Kroemer, C. H. Lampert, and J. Peters, "Learning dynamic tactile sensing with robust vision-based training," IEEE Transactions on Robotics, vol. 27, no. 3, pp. 545-557, 2011.

[101] J. Hoelscher, J. Peters, and T. Hermans, "Evaluation of tactile feature extraction for interactive object recognition," in IEEE-RAS 15th International Conference on Humanoid Robots (Humanoids), pp. 310-317, IEEE, 2015.

[102] J. Sinapov, V. Sukhoy, R. Sahai, and A. Stoytchev, "Vibrotactile recognition and categorization of surfaces by a humanoid robot," IEEE Transactions on Robotics, vol. Vol.27, no. No.3, pp. 488-497, 2011. 
[103] J. Wade, T. Bhattacharjee, and C. C. Kemp, "Force and thermal sensing with a fabric-based skin," in See, Touch and Hear: 2nd Workshop on multimodal sensor-based robot control for HRI and soft manipulation, IEEE/RSJ International Conference on Intelligent Robots and Systems (IROS), 2016.

[104] D. Xu, G. E. Loeb, and J. A. Fishel, "Tactile identification of objects using bayesian exploration," in IEEE International Conference on Robotics and Automation (ICRA), pp. 3056-3061, IEEE, 2013.

[105] Y. Ding, J. Bonse, R. Andre, and U. Thomas, "In-hand grasping pose estimation using particle filters in combination with haptic rendering models," International Journal of Humanoid Robotics, vol. 15, no. 01, p. 1850002, 2018.

[106] M. Pfanne and M. Chalon, "Ekf-based in-hand object localization from joint position and torque measurements," in IEEE/RSJ International Conference on Intelligent Robots and Systems (IROS), pp. 2464-2470, IEEE, 2017.

[107] M. C. Koval, M. R. Dogar, N. S. Pollard, and S. S. Srinivasa, "Pose estimation for contact manipulation with manifold particle filters," in IEEE/RSJ International Conference on Intelligent Robots and Systems (IROS), pp. 4541-4548, IEEE, 2013.

[108] S. Li, S. Lyu, and J. Trinkle, "State estimation for dynamic systems with intermittent contact," in IEEE International Conference on Robotics and Automation (ICRA), pp. 3709-3715, IEEE, 2015.

[109] A. Petrovskaya, O. Khatib, S. Thrun, and A. Y. Ng, "Bayesian estimation for autonomous object manipulation based on tactile sensors," in IEEE International Conference on Robotics and Automation (ICRA), pp. 707-714, IEEE, 2006.

[110] A. Bierbaum, M. Rambow, T. Asfour, and R. Dillmann, "A potential field approach to dexterous tactile exploration of unknown objects," in IEEE-RAS 15th International Conference on Humanoid Robots (Humanoids), pp. 360-366, IEEE, 2008.

[111] M. Kaboli, D. Feng, K. Yao, P. Lanillos, and G. Cheng, "A tactilebased framework for active object learning and discrimination using multimodal robotic skin," IEEE Robotics and Automation Letters, vol. 2, no. 4, pp. 2143-2150, 2017.

[112] M. Kaboli, K. Yao, D. Feng, and G. Cheng, "Tactile-based active object discrimination and target object search in an unknown workspace," Autonomous Robots, vol. 42, no. 3, pp. 1573-7527, 2018.

[113] G. Vezzani, U. Pattacini, G. Battistelli, L. Chisci, and L. Natale, "Memory unscented particle filter for 6-dof tactile localization," IEEE Transactions on Robotics, vol. 33, no. 5, pp. 1139-1155, 2017.

[114] L. Zhang and J. C. Trinkle, "The application of particle filtering to grasping acquisition with visual occlusion and tactile sensing," in IEEE International Conference on Robotics and Automation (ICRA), pp. 3805-3812, IEEE, 2012

[115] M. Moll and M. A. Erdmann, "Reconstructing shape from motion using tactile sensors," in IEEE/RSJ International Conference on Intelligent Robots and Systems (IROS), vol. 2, pp. 692-700, IEEE, 2001.

[116] J. Ilonen, J. Bohg, and V. Kyrki, "Three-dimensional object reconstruction of symmetric objects by fusing visual and tactile sensing," International Journal of Robotics Research, vol. 33, no. 2, pp. 321341,2014

[117] M. Bjorkman, Y. Bekiroglu, V. Hogman, and D. Kragic, "Enhancing visual perception of shape through tactile glances," in IEEE/RSJ International Conference on Intelligent Robots and Systems (IROS), pp. 3180-3186, IEEE, 2013.

[118] S. Wang, J. Wu, X. Sun, W. Yuan, W. T. Freeman, J. B. Tenenbaum, and E. H. Adelson, "3d shape perception from monocular vision, touch, and shape priors," in IEEE/RSJ International Conference on Intelligent Robots and Systems (IROS), pp. 1606-1613, IEEE, 2018.

[119] T. Matsubara, K. Shibata, and K. Sugimoto, "Active touch point selection with travel cost in tactile exploration for fast shape estimation of unknown objects," in IEEE/ASME International Conference on Advanced Intelligent Mechatronics (AIM), pp. 1115-1120, IEEE, 2016.

[120] Z. Yi, R. Calandra, F. Veiga, H. van Hoof, T. Hermans, Y. Zhang, and J. Peters, "Active tactile object exploration with gaussian processes," in IEEE/RSJ International Conference on Intelligent Robots and Systems (IROS), pp. 4925-4930, IEEE, 2016.

[121] N. Jamali, C. Ciliberto, L. Rosasco, and L. Natale, "Active perception: Building objects' models using tactile exploration," in IEEE-RAS 15th International Conference on Humanoid Robots (Humanoids), pp. 179185, IEEE, 2016.

[122] C. G. Atkeson, C. H. An, and J. M. Hollerbach, "Rigid body load identification for manipulators," in IEEE Conference on Decision and Control (CDC), pp. 996-1002, IEEE, 1985

[123] M. Kaboli, K. Yao, and G. Cheng, "Tactile-based manipulation of deformable objects with dynamic center of mass," in IEEE-RAS 15th
International Conference on Humanoid Robots (Humanoids), pp. 752757, IEEE, 2016.

[124] K. Yao, M. Kaboli, and G. Cheng, "Tactile-based object center of mass exploration and discrimination," in IEEE-RAS 15th International Conference on Humanoid Robots (Humanoids), pp. 876-881, IEEE, 2017.

[125] Y. Yu, K. Fukuda, and S. Tsujio, "Estimation of mass and center of mass of graspless and shape-unknown object," in IEEE International Conference on Robotics and Automation (ICRA), vol. 4, pp. 2893-2898, IEEE, 1999

[126] D. Kanoulas, J. Lee, D. G. Caldwell, and N. G. Tsagarakis, "Centerof-mass-based grasp pose adaptation using $3 \mathrm{~d}$ range and force/torque sensing," International Journal of Humanoid Robotics, vol. 15, no. 04, p. $1850013,2018$.

[127] D. Kubus, T. Kroger, and F. M. Wahl, "On-line rigid object recognition and pose estimation based on inertial parameters," in IEEE/RSJ International Conference on Intelligent Robots and Systems (IROS), pp. 1402-1408, IEEE, 2007.

[128] Y. Yu, T. Arima, and S. Tsujio, "Estimation of object inertia parameters on robot pushing operation," IEEE International Conference on Robotics and Automation (ICRA), pp. 1657-1662, 2005.

[129] S. Chitta, J. Sturm, M. Piccoli, and W. Burgard, "Tactile sensing for mobile manipulation," IEEE Transactions on Robotics, vol. 27, no. 3, pp. 558-568, 2011.

[130] H. P. Saal, J.-A. Ting, and S. Vijayakumar, "Active estimation of object dynamics parameters with tactile sensors," in IEEE/RSJ International Conference on Intelligent Robots and Systems (IROS), pp. 916-921, IEEE, 2010.

[131] S. Clarke, T. Rhodes, C. G. Atkeson, and O. Kroemer, "Learning audio feedback for estimating amount and flow of granular material," in Conference on Robot Learning (CORL), pp. 529-550, 2018.

[132] R. S. Johansson, J. R. Flanagan, and R. S. Johansson, "Sensory control of object manipulation," Sensorimotor Control of Grasping: Physiology and Pathophysiology, pp. 141-160, 2009.

[133] Y. Yu, T. Kiyokawa, and S. Tsujio, "Estimation of mass and center of mass of unknown and graspless cylinder-like object," International Journal of Information Acquisition, vol. 1, no. 01, pp. 47-55, 2004.

[134] Y. Chebotar, K. Hausman, Z. Su, G. S. Sukhatme, and S. Schaal, "Self-supervised regrasping using spatio-temporal tactile features and reinforcement learning," in IEEE/RSJ International Conference on Intelligent Robots and Systems (IROS), pp. 1960-1966, IEEE, 2016.

[135] R. Calandra, A. Owens, D. Jayaraman, J. Lin, W. Yuan, J. Malik, E. H. Adelson, and S. Levine, "More than a feeling: Learning to grasp and regrasp using vision and touch," IEEE Robotics and Automation Letters, vol. 3, no. 4, pp. 3300-3307, 2018.

[136] R. D. Howe and M. R. Cutkosky, "Practical force-motion models for sliding manipulation," International Journal of Robotics Research, vol. 15 , no. 6, pp. 557-572, 1996.

[137] Q. Li, M. Meier, R. Haschke, H. Ritter, and B. Bolder, "Rotary object dexterous manipulation in hand: a feedback-based method," International Journal of Mechatronics and Automation, vol. 3, no. 1, pp. $36-47,2013$.

[138] H. Zhang and N. N. Chen, "Control of contact via tactile sensing," IEEE Transactions on Robotics and Automation, vol. 16, no. 5, pp. 482-495, 2000.

[139] Y. Chebotar, O. Kroemer, and J. Peters, "Learning robot tactile sensing for object manipulation," in IEEE/RSJ International Conference on Intelligent Robots and Systems (IROS), pp. 3368-3375, IEEE, 2014.

[140] D. Kappler, P. Pastor, M. Kalakrishnan, W. Manue, and S. Schaal, "Data-driven online decision making for autonomous manipulation," Robotics: Science and Systems (RSS), 2015.

[141] N. F. Lepora, K. Aquilina, and L. Cramphorn, "Exploratory tactile servoing with active touch," IEEE Robotics and Automation Letters, vol. 2, no. 2, pp. 1156-1163, 2017.

[142] N. F. Lepora, A. Church, C. De Kerckhove, R. Hadsell, and J. Lloyd, "From pixels to percepts: Highly robust edge perception and contour following using deep learning and an optical biomimetic tactile sensor," IEEE Robotics and Automation Letters, vol. 4, no. 2, pp. 2101-2107, 2019

[143] Z. Su, O. Kroemer, G. E. Loeb, G. S. Sukhatme, and S. Schaal, "Learning to switch between sensorimotor primitives using multimodal haptic signals," in International Conference on Simulation of Adaptive Behavior (SAB), pp. 170-182, Springer, 2016.

[144] F. Veiga, D. Notz, T. Hesse, and J. Peters, "Tactile based forward modeling for contact location control," Workshop on Tactile Sensing for Manipulation, Robotics: Science and Systems, 2017. 
[145] P. Pastor, M. Kalakrishnan, S. Chitta, E. Theodorou, and S. Schaal, "Skill learning and task outcome prediction for manipulation," in IEEE International Conference on Robotics and Automation (ICRA), pp. 3828-3834, IEEE, 2011.

[146] H. Dang and P. K. Allen, "Learning grasp stability," in IEEE International Conference on Robotics and Automation (ICRA), pp. 2392-2397, IEEE, 2012.

[147] M. Madry, L. Bo, D. Kragic, and D. Fox, "St-hmp: Unsupervised spatio-temporal feature learning for tactile data," in IEEE International Conference on Robotics and Automation (ICRA), pp. 2262-2269, IEEE, 2014.

[148] Y. Bekiroglu, J. Laaksonen, J. A. Jorgensen, V. Kyrki, and D. Kragic, "Assessing grasp stability based on learning and haptic data," IEEE Transactions on Robotics, vol. 27, no. 3, pp. 616-629, 2011.

[149] G. J. Maeda, G. Neumann, M. Ewerton, R. Lioutikov, O. Kroemer, and J. Peters, "Probabilistic movement primitives for coordination of multiple human-robot collaborative tasks," Autonomous Robots, vol. 41, no. 3, pp. 593-612, 2017.

[150] O. Kroemer, S. Leischnig, S. Luettgen, and J. Peters, "A kernel-based approach to learning contact distributions for robot manipulation tasks," Autonomous Robots, vol. 42, no. 3, pp. 581-600, 2018.

[151] H. van Hoof, T. Hermans, G. Neumann, and J. Peters, "Learning robot in-hand manipulation with tactile features," in IEEE-RAS 15th International Conference on Humanoid Robots (Humanoids), pp. 121127, 2015.

[152] M. Andrychowicz, B. Baker, M. Chociej, R. Jozefowicz, B. McGrew, J. Pachocki, A. Petron, M. Plappert, G. Powell, A. Ray, et al., "Learning dexterous in-hand manipulation," IJRR, 2019.

[153] Z. Su, O. Kroemer, G. E. Loeb, G. S. Sukhatme, and S. Schaal, "Learning manipulation graphs from demonstrations using multimodal sensory signals," in IEEE International Conference on Robotics and Automation (ICRA), IEEE, 2018.

[154] H. van Hoof, N. Chen, M. Karl, P. van der Smagt, and J. Peters, "Stable reinforcement learning with autoencoders for tactile and visual data," in IEEE/RSJ International Conference on Intelligent Robots and Systems (IROS), pp. 3928-3934, IEEE, 2016.

[155] Y. Gao, L. A. Hendricks, K. J. Kuchenbecker, and T. Darrell, "Deep learning for tactile understanding from visual and haptic data," in IEEE International Conference on Robotics and Automation (ICRA), pp. 536-543, IEEE, 2016.

[156] S. Ottenhaus, M. Miller, D. Schiebener, N. Vahrenkamp, and T. Asfour, "Local implicit surface estimation for haptic exploration," in IEEERAS 15th International Conference on Humanoid Robots (Humanoids), pp. 850-856, IEEE, 2016.

[157] N. F. Lepora, U. Martinez-Hernandez, and T. J. Prescott, "Active bayesian perception for simultaneous object localization and identification.," in Robotics: Science and Systems (RSS), 2013.

[158] N. Sommer and A. Billard, "Multi-contact haptic exploration and grasping with tactile sensors," Robotics and Autonomous Systems, vol. 85, pp. 48-61, 2016.

[159] L. Cramphorn, B. Ward-Cherrier, and N. F. Lepora, "Tactile manipulation with biomimetic active touch," in 2016 IEEE International Conference on Robotics and Automation (ICRA), pp. 123-129, IEEE, 2016

[160] G. Sutanto, N. Ratliff, B. Sundaralingam, Y. Chebotar, Z. Su, A. Handa, and D. Fox, "Learning latent space dynamics for tactile servoing," in 2019 International Conference on Robotics and Automation (ICRA), pp. 3622-3628, IEEE, 2019.

[161] D. J. M. M. C. F. R. C. Stephen Tian, Frederik Ebert and S. Levine, "Manipulation by feel: Touch-based control with deep predictive models," in IEEE International Conference on Robotics and Automation (ICRA), IEEE, 2019

[162] M. Regoli, U. Pattacini, G. Metta, and L. Natale, "Hierarchical grasp controller using tactile feedback," in Humanoid Robots (Humanoids), 2016 IEEE-RAS 16th International Conference on, pp. 387-394, IEEE, 2016.

[163] R. Platt Jr, A. H. Fagg, and R. A. Grupen, "Null-space grasp control: Theory and experiments," IEEE Transactions on Robotics, vol. 26, no. 2, pp. 282-295, 2010.

[164] M. Li, H. Yin, K. Tahara, and A. Billard, "Learning object-level impedance control for robust grasping and dexterous manipulation," in Robotics and Automation (ICRA), 2014 IEEE International Conference on, pp. 6784-6791, IEEE, 2014.

[165] J. Gandarias, J. Gómez-de Gabriel, and A. García-Cerezo, "Enhancing perception with tactile object recognition in adaptive grippers for human-robot interaction," Sensors, vol. 18, no. 3, p. 692, 2018.
[166] A. Schmitz, Y. Bansho, K. Noda, H. Iwata, T. Ogata, and S. Sugano, "Tactile object recognition using deep learning and dropout," in 2014 IEEE-RAS International Conference on Humanoid Robots, pp. 10441050, IEEE, 2014.

[167] L. Cao, R. Kotagiri, F. Sun, H. Li, W. Huang, and Z. M. M. Aye, "Efficient spatio-temporal tactile object recognition with randomized tiling convolutional networks in a hierarchical fusion strategy," in Thirtieth AAAI Conference on Artificial Intelligence, 2016.

[168] S. Dragiev, M. Toussaint, and M. Gienger, "Uncertainty aware grasping and tactile exploration," in Robotics and Automation (ICRA), 2013 IEEE International Conference on, pp. 113-119, IEEE, 2013.

[169] H. Dang and P. K. Allen, "Stable grasping under pose uncertainty using tactile feedback," Autonomous Robots, vol. 36, no. 4, pp. 309-330, 2014.

[170] M. U. W. Y. J. L. E. H. A. Roberto Calandra, Andrew Owens and S. Levine, "The feeling of success: Does touch sensing help predict grasp outcomes?", in Conference on Robot Learning (CORL), 2017.

[171] A. Delgado, C. A. Jara, and F. Torres, "Adaptive tactile control for in-hand manipulation tasks of deformable objects," The International Journal of Advanced Manufacturing Technology, vol. 91, no. 9-12, pp. 4127-4140, 2017.

[172] Q. Li, R. Haschke, B. Bolder, and H. Ritter, "Grasp point optimization by online exploration of unknown object surface," in 2012 12th IEEE-RAS International Conference on Humanoid Robots (Humanoids 2012), pp. 417-422, IEEE, 2012.

[173] A. B. Cole, J. E. Hauser, and S. S. Sastry, "Kinematics and control of multifingered hands with rolling contact," IEEE Transactions on Automatic Control, vol. 34, no. 4, pp. 398-404, 1989.

[174] C. A. Jara, J. Pomares, F. A. Candelas, and F. Torres, "Control framework for dexterous manipulation using dynamic visual servoing and tactile sensors feedback," Sensors, vol. 14, no. 1, pp. 1787-1804, 2014

[175] Y. Kurita, J. Ueda, Y. Matsumoto, and T. Ogasawara, "Cpg-based manipulation: Generation of rhythmic finger gaits from human observation," in IEEE International Conference on Robotics and Automation (ICRA), vol. 2, pp. 1209-1214, IEEE, 2004.

[176] R. Platt, A. H. Fagg, and R. A. Grupen, "Manipulation gaits: Sequences of grasp control tasks," in IEEE International Conference on Robotics and Automation (ICRA), vol. 1, pp. 801-806, IEEE, 2004.

[177] J. A. C. Ramón, F. T. Medina, and V. Perdereau, "Finger readjustment algorithm for object manipulation based on tactile information," International Journal of Advanced Robotic Systems, vol. 10, no. 1, p. 9 , 2013.

[178] B. Ward-Cherrier, N. Rojas, and N. F. Lepora, "Model-free precise inhand manipulation with a 3d-printed tactile gripper," IEEE Robotics and Automation Letters, vol. 2, no. 4, pp. 2056-2063, 2017.

[179] V. Kumar, A. Gupta, E. Todorov, and S. Levine, "Learning dexterous manipulation policies from experience and imitation," Robotics: Science and Systems (RSS), 2018.

[180] Q. Li, R. Haschke, and H. Ritter, "Learning a tool's homogeneous transformation by tactile-based interaction," in IEEE-RAS 15th International Conference on Humanoid Robots (Humanoids), pp. 416-421, IEEE, 2016.

[181] Q. Li, A. Ückermann, R. Haschke, and H. Ritter, "Estimating an articulated tool's kinematics via visuo-tactile based robotic interactive manipulation," in 2018 IEEE/RSJ International Conference on Intelligent Robots and Systems (IROS), pp. 6938-6944, IEEE, 2018.

[182] H. Hoffmann, Z. Chen, D. Earl, D. Mitchell, B. Salemi, and J. Sinapov, "Adaptive robotic tool use under variable grasps," Robotics and Autonomous Systems, vol. 62, no. 6, pp. 833-846, 2014.

[183] G. Sutanto, Z. Su, S. Schaal, and F. Meier, "Learning sensor feedback models from demonstrations via phase-modulated neural networks," in 2018 IEEE International Conference on Robotics and Automation (ICRA), pp. 1142-1149, IEEE, 2018.

[184] T. Okatani, H. Takahashi, T. Takahata, and I. Shimoyama, "Evaluation of ground slippery condition during walk of bipedal robot using mems slip sensor," in Micro Electro Mechanical Systems (MEMS), 2017 IEEE 30th International Conference on, pp. 1033-1035, IEEE, 2017.

[185] K. Walas, "Terrain classification and negotiation with a walking robot," Journal of Intelligent Robotic Systems, vol. 78, no. 3-4, pp. 401-423, 2015.

[186] A. Kalamdani, C. Messom, and M. Siegel, "Tactile sensing by the sole of the foot: part i: apparatus and initial experiments toward obtaining dynamic pressure maps useful for stabilizing standing, walking, and running of humanoid robots," in IEEE International Workshop on Haptic Audio Visual Environments and their Applications (HAVE), pp. 147-151, IEEE, 2006 
[187] K. Suwanratchatamanee, M. Matsumoto, and S. Hashimoto, "Walking on the slopes with tactile sensing system for humanoid robot," in International Conference on Control Automation and Systems (ICCAS), pp. 350-355, IEEE, 2010.

[188] K. Hauser, "Bayesian tactile exploration for compliant docking with uncertain shapes," in Robotics: Science and Systems (RSS), 2018.

[189] C. A. Brooks and K. D. Iagnemma, "Self-supervised classification for planetary rover terrain sensing," in IEEE Aerospace Conference (AeroConf), pp. 1-9, IEEE, 2007.

[190] A. Valada, L. Spinello, and W. Burgard, "Deep feature learning for acoustics-based terrain classification," in Robotics Research, pp. 2137, Springer, 2018.

[191] J. Libby and A. J. Stentz, "Using sound to classify vehicle-terrain interactions in outdoor environments," in IEEE International Conference on Robotics and Automation (ICRA), pp. 3559-3566, IEEE, 2012.

[192] A. Cirillo, P. Cirillo, G. De Maria, C. Natale, and S. Pirozzi, "A distributed tactile sensor for intuitive human-robot interfacing," Journal of Sensors, vol. 2017, 2017

[193] P. M. Grice, M. D. Killpack, A. Jain, S. Vaish, J. Hawke, and C. C. Kemp, "Whole-arm tactile sensing for beneficial and acceptable contact during robotic assistance," in IEEE International Conference on Rehabilitation Robotics (ICORR), pp. 1-8, IEEE, 2013.

[194] A. G. Eguíluz, I. Rañó, S. Coleman, and T. McGinnity, "Reliable robotic handovers through tactile sensing," Autonomous Robots, pp. 1$15,2019$.

[195] K. Yokoyama, H. Handa, T. Isozumi, Y. Fukase, K. Kaneko, F. Kanehiro, Y. Kawai, F. Tomita, and H. Hirukawa, "Cooperative works by a human and a humanoid robot," in IEEE International Conference on Robotics and Automation (ICRA), vol. 3, pp. 2985-2991, IEEE, 2003.

[196] Z. Erickson, H. M. Clever, G. Turk, C. K. Liu, and C. C. Kemp, "Deep haptic model predictive control for robot-assisted dressing," in IEEE International Conference on Robotics and Automation (ICRA), pp. 1-8, IEEE, 2018.

[197] F. DallaLibera, F. Basoeki, T. Minato, H. Ishiguro, and E. Menegatti, "Teaching by touching: Interpretation of tactile instructions for motion development," in IEEE/RSJ International Conference on Intelligent Robots and Systems (IROS), pp. 3480-3487, IEEE, 2011.

[198] J. Bohg, A. Morales, T. Asfour, and D. Kragic, "Data-driven grasp synthesisa survey," IEEE Transactions on Robotics, vol. 30, no. 2, pp. 289-309, 2013.

[199] M. A. Roa and R. Suárez, "Grasp quality measures: review and performance," Autonomous Robots, vol. 38, no. 1, pp. 65-88, 2015.

[200] S. Dragiev, M. Toussaint, and M. Gienger, "Gaussian process implicit surfaces for shape estimation and grasping," IEEE International Conference on Robotics and Automation (ICRA), pp. 2845-2850, 2011.

[201] M. Costanzo, G. De Maria, and C. Natale, "Slipping control algorithms for object manipulation with sensorized parallel grippers," in 2018 IEEE International Conference on Robotics and Automation (ICRA), pp. 7455-7461, IEEE, 2018.

[202] A. Cirillo, P. Cirillo, G. De Maria, C. Natale, and S. Pirozzi, "Control of linear and rotational slippage based on six-axis force/tactile sensor," in 2017 IEEE International Conference on Robotics and Automation (ICRA), pp. 1587-1594, IEEE, 2017.

[203] D. Gunji, T. Araki, A. Namiki, A. Ming, and M. Shimojo, "Grasping force control of multi-fingered robot hand based on slip detection using tactile sensor," Journal of the Robotics Society of Japan, vol. 25, no. 6 , pp. 970-978, 2007.

[204] N. Y. Chong, D. Choi, and I. H. Suh, "A generalized motion/force planning strategy for multifingered hands using both rolling and sliding contacts," in IEEE/RSJ International Conference on Intelligent Robots and Systems (IROS), vol. 1, pp. 113-120, IEEE, 1993.

[205] L. Han and J. C. Trinkle, "Dextrous manipulation by rolling and finger gaiting," in IEEE International Conference on Robotics and Automation (ICRA), vol. 1, pp. 730-735, IEEE, 1998.

[206] Y. Bai and C. K. Liu, "Dexterous manipulation using both palm and fingers," in IEEE International Conference on Robotics and Automation (ICRA), pp. 1560-1565, IEEE, 2014.

[207] T. Ishihara, A. Namiki, M. Ishikawa, and M. Shimojo, "Dynamic pen spinning using a high-speed multifingered hand with high-speed tactile sensor," in IEEE-RAS 15th International Conference on Humanoid Robots (Humanoids), pp. 258-263, IEEE, 2006.

[208] Y. Karayiannidis, C. Smith, D. Kragic, et al., "Adaptive control for pivoting with visual and tactile feedback," in 2016 IEEE International Conference on Robotics and Automation (ICRA), pp. 399-406, IEEE, 2016
[209] E. D. Engeberg and S. G. Meek, "Adaptive sliding mode control for prosthetic hands to simultaneously prevent slip and minimize deformation of grasped objects," IEEE/ASME Transactions on Mechatronics, vol. 18 , no. 1, pp. 376-385, 2011.

[210] J. Shi, J. Z. Woodruff, P. B. Umbanhowar, and K. M. Lynch, "Dynamic in-hand sliding manipulation," IEEE Transactions on Robotics, vol. 33 , no. 4, pp. 778-795, 2017.

[211] T. Okada, "Object-handling system for manual industry," IEEE Transactions on Systems, Man, and Cybernetics, vol. 9, no. 2, pp. 79-89, 1979.

[212] A. L. Ciancio, L. Zollo, E. Guglielmelli, D. Caligiore, and G. Baldassarre, "Hierarchical reinforcement learning and central pattern generators for modeling the development of rhythmic manipulation skills," in IEEE International Conference on Development and Learning (ICDL), vol. 2, pp. 1-8, IEEE, 2011.

[213] C. C. Kemp and A. Edsinger, "Robot manipulation of human tools: Autonomous detection and control of task relevant features," in IEEE International Conference on Development and Learning (ICDL), vol. 42, 2006.

[214] M. A. Hoepflinger, C. D. Remy, M. Hutter, L. Spinello, and R. Siegwart, "Haptic terrain classification for legged robots," in IEEE International Conference on Robotics and Automation (ICRA), pp. 2828-2833, IEEE, 2010.

[215] S. Kajita, H. Hirukawa, K. Harada, and K. Yokoi, Introduction to Humanoid Robotics, vol. 101. Springer, 2014.

[216] A. G. Eguíluz, I. Rano, S. A. Coleman, and T. M. McGinnity, "Reliable object handover through tactile force sensing and effort control in the shadow robot hand," in IEEE International Conference on Robotics and Automation (ICRA), pp. 372-377, IEEE, 2017.

[217] B. D. R. G. (BDR), "Touch-sensitive skin for collaborative industrial robots." https://cordis.europa.eu/project/rcn/213689/factsheet/en, 2019. [Online; accessed 7-Nov-2019].

[218] A. Cirillo, F. Ficuciello, C. Natale, S. Pirozzi, and L. Villani, "A conformable force/tactile skin for physical human-robot interaction," IEEE Robotics and Automation Letters, vol. 1, no. 1, pp. 41-48, 2015.

[219] M. Kaboli, A. Long, and G. Cheng, "Humanoids learn touch modalities identification via multi-modal robotic skin and robust tactile descriptors," Advanced Robotics, vol. 29, no. 21, pp. 1411-1425, 2015.

[220] Y. Chen, M. Yu, H. A. Bruck, and E. Smela, "Compliant multi-layer tactile sensing for enhanced identification of human touch," Smart Materials and Structures, vol. 27, no. 12, p. 125009, 2018.

[221] D. Hughes, J. Lammie, and N. Correll, "A robotic skin for collision avoidance and affective touch recognition," IEEE Robotics and $\mathrm{Au}$ tomation Letters, vol. 3, no. 3, pp. 1386-1393, 2018.

[222] T. Shibata and K. Tanie, "Physical and affective interaction between human and mental commit robot," in IEEE International Conference on Robotics and Automation (ICRA), vol. 3, pp. 2572-2577, IEEE, 2001.

[223] H. Iwata and S. Sugano, "Whole-body covering tactile interface for human robot coordination," in IEEE International Conference on Robotics and Automation (ICRA), vol. 4, pp. 3818-3824, IEEE, 2002. 